\title{
AN ESSENTIAL PROPERTY OF THE FOURIER TRANSFORMS OF DISTRIBUTION FUNCTIONS
}

\author{
EUGENE LUKACS
}

Fourier transforms of distribution functions are an important tool in the theory of probability because they have certain properties. These properties are expressed in the uniqueness theorem, the convolution theorem, and the continuity theorem (cf. [1, pp. 93, 96, 188]). This note deals with the question of whether there are any other integral transforms of distribution functions which have these properties.

We consider an integral transform

$$
f(s)=\int_{-\infty}^{+\infty} K(s, t) d F(t)
$$

of a distribution function $F(t) .{ }^{1}$ We denote in the following by $F_{1}(t), F_{2}(t)$ distribution functions and by $f_{1}(s), f_{2}(s)$ their respective transforms by $K(s, t)$.

We make the following assumptions:

(I) The kernel $K(s, t)$ is a complex-valued function defined for all values of the real variables $s$ and $t$ and is bounded and $B$-measurable in $t$.

(II) (Uniqueness) $f_{1}(s) \equiv f_{2}(s)$ if and only if $F_{1}(t) \equiv F_{2}(t)$.

(III) (Convolution) If we let $F(x) \equiv F_{1} * F_{2}=\int_{-\infty}^{+\infty} F_{1}(x-t) d F_{2}(t)=$ $\int_{-\infty}^{+\infty} F_{2}(x-t) d F_{1}(t)$ be the convolution of two distribution functions, then

$$
f(s)=f_{1}(s) f_{2}(s) .
$$

The purpose of this note is to prove: $A$ kernel $K(s, t)$ satisfies assumptions (I), (II), and (III) if and only if it has the form

$$
K(s, t)=e^{i t A(s)}
$$

where $A(s)$ is a real-valued function which assumes all values of a set which is dense in the real line. Moreover, $A\left(s_{1}\right) \neq A\left(s_{2}\right)$ if $s_{1} \neq s_{2}$.

We see from assumption (I) that every distribution function $F(t)$ has a transform given by (1). We write assumption (III) in terms of the kernel and obtain

Presented to the Society, December 26, 1951; received by the editors October 4, 1951.

${ }^{1}$ As customary in probability theory we say that a nondecreasing function $F(t)$ is a distribution function if it is everywhere continuous to the right and if $F(-\infty)$ $=0, F(+\infty)=1$. 


$$
\begin{aligned}
\int_{-\infty}^{+\infty} K(s, v) d v \int_{-\infty}^{+\infty} F_{1}(v-u) d F_{2}(u) & \\
& =\int_{-\infty}^{+\infty} K(s, t) d F_{1}(t) \int_{-\infty}^{+\infty} K(s, u) d F_{2}(u) \\
& =\int_{-\infty}^{+\infty} \int_{-\infty}^{\infty} K(s, t) K(s, u) d F_{1}(t) d F_{2}(u)
\end{aligned}
$$

On the other hand

$$
\int_{-\infty}^{+\infty} K(s, v) d_{v} \int_{-\infty}^{\infty} F_{1}(v-u) d F_{2}(u)=\int_{-\infty}^{+\infty} \int_{-\infty}^{+\infty} K(s, t+u) d F_{1}(t) d F_{2}(u)
$$

so that

$$
\begin{aligned}
\int_{-\infty}^{+\infty} \int_{-\infty}^{+\infty} K(s, t+u) d F_{1}(t) d F_{2}(u) & \\
= & \int_{-\infty}^{+\infty} \int_{-\infty}^{+\infty} K(s, t) K(s, u) d F_{1}(t) d F_{2}(u) .
\end{aligned}
$$

Denote by $\epsilon(x)$ the degenerate distribution which has a saltus of 1 at $x=0$, that is

$$
\epsilon(x)= \begin{cases}0 & \text { if } x<0, \\ 1 & \text { if } x \geqq 0 .\end{cases}
$$

Then $\epsilon(x-a)$ is a distribution with a single saltus of 1 at the point $x=a$. We apply assumption (III) to the distribution functions $F_{1}(x)$ $=\epsilon(x-\eta)$ and $F_{2}(x)=[\epsilon(x)+\epsilon(x-\xi)] / 2$ where $\xi$ and $\eta$ are arbitrary real numbers. We see then easily that

$$
K(s, \eta)+K(s, \eta+\xi)=K(s, \eta)[K(s, 0)+K(s, \xi)]
$$

for any real $\xi$ and $\eta$, in particular we obtain for $\xi=0$

$$
2 K(s, \eta)=2 K(s, 0) K(s, \eta) \text {. }
$$

Therefore (5) reduces to

$$
K(s, \eta+\xi)=K(s, \eta) K(s, \xi) .
$$

Since $K(s, t)$ is measurable in $t$, every solution of this equation is of the form $K(s, t)=e^{t \rho(s)} .^{2}$

Let $\rho(s)=B(s)+i A(s)$; then $|K(s, t)|=e^{t B(s)}$.

Since $K(s, t)$ is bounded we have $B(s) \equiv 0$. The kernel has therefore

See [2, pp. 116-118]. 
the form

$$
K(s, t)=e^{i t A(s)} .
$$

Hence the transform of $F(t)$ is given by

$$
f(s)=\int_{-\infty}^{\infty} e^{i t A(s)} d F(t) .
$$

The characteristic function (Fourier transform) of the distribution $F(t)$ is

$$
\phi(u)=\int_{-\infty}^{\infty} e^{i t u} d F(t)
$$

From (8) and (9) it is seen that

$$
\phi[A(s)]=f(s) .
$$

From the theory of characteristic functions (see $[1$, p. 94]) we see that $F_{1}(t) \equiv F_{2}(t)$ if and only if $\phi_{1}(u) \equiv \phi_{2}(u)$ for all values of $u$; the knowledge of the characteristic functions over a finite interval is not sufficient for the determination of $F(t)$. It follows therefore from (9.1) and assumption (II) that $A(s)$ must assume all values of a dense set between $-\infty$ and $+\infty$. We finally show by an indirect proof that $A\left(s_{1}\right) \neq A\left(s_{2}\right)$ if $s_{1} \neq s_{2}$. If this would not be the case the distributions $\epsilon\left(x-s_{1}\right)$ and $\epsilon\left(x-s_{2}\right)$ would have the same transforms, namely $e^{i t A\left(s_{1}\right)}=e^{i t A\left(s_{2}\right)}$ in contradiction to assumption (II).

We still have to show that the representation (3) of the kernel is sufficient to assure the validity of (I), (II), and (III). This is immediately seen for (I), the proof of (II) can be carried out in the customary manner with the aid of Weierstrass' approximation theorem (see [3]). We see finally from (4) and (7) that assumption (III) is also satisfied.

It is of interest to note that the uniqueness theorem and the convolution theorem determine the form of the kernel, without using the continuity theorem.

\section{REFERENCES}

1. H. Cramér, Mathematical methods of statistics, Princeton University Press, 1946.

2. H. Hahn and A. Rosenthal, Set functions, Albuquerque, The University of New Mexico Press, 1948.

3. A. Wintner, The Fourier transforms of probability distributions, Baltimore, 1947.

NATional BUREAU OF Standards 